\title{
Jung bleiben mit Omega-3-Fettsäuren
}

\section{Autor: Volker Schmiedel}

\section{ZUSAMMENFASSUNG}

Stille Entzündung ist ein Risikofaktor für das Altern. Alle Faktoren, die dazu beitragen, Entzündungen zu reduzieren, sollten daher auch den Alterungsprozess verlangsamen können. Medikamente wie Kortison oder NSAR kommen wegen zahlreicher Nebenwirkungen allerdings nicht in Frage. Die antiinflammatorische Wirkung von Omega-3-Fettsäuren ist seit langer Zeit bekannt. Gibt es auch Nachweise, dass AItersbeschwerden wie Demenz, Sarkopenie oder Hautalterung durch Omega-3-Fettsäuren aufgehalten werden können? Der Artikel gibt eine Übersicht über die wichtigsten Studien.

\section{ABSTRACT}

Silent inflammation is a risk factor for aging. Therefore, all factors contributing to the reduction of inflammations should also be able to slow down the aging process. However, drugs such as cortisone or NSAIDs are out of the question because of numerous side effects. The antiinflammatory effect of omega-3 fatty acids has been known for a long time. Is there also proof, that complaints of old age such as dementia, sarcopenia or skin aging can be stopped by omega- 3 fatty acids? The article provides an overview of the most important studies.

\section{Keywords}

Silent inflammation, omega-3 fatty acids, aging, dementia, sarcopenia, skin aging

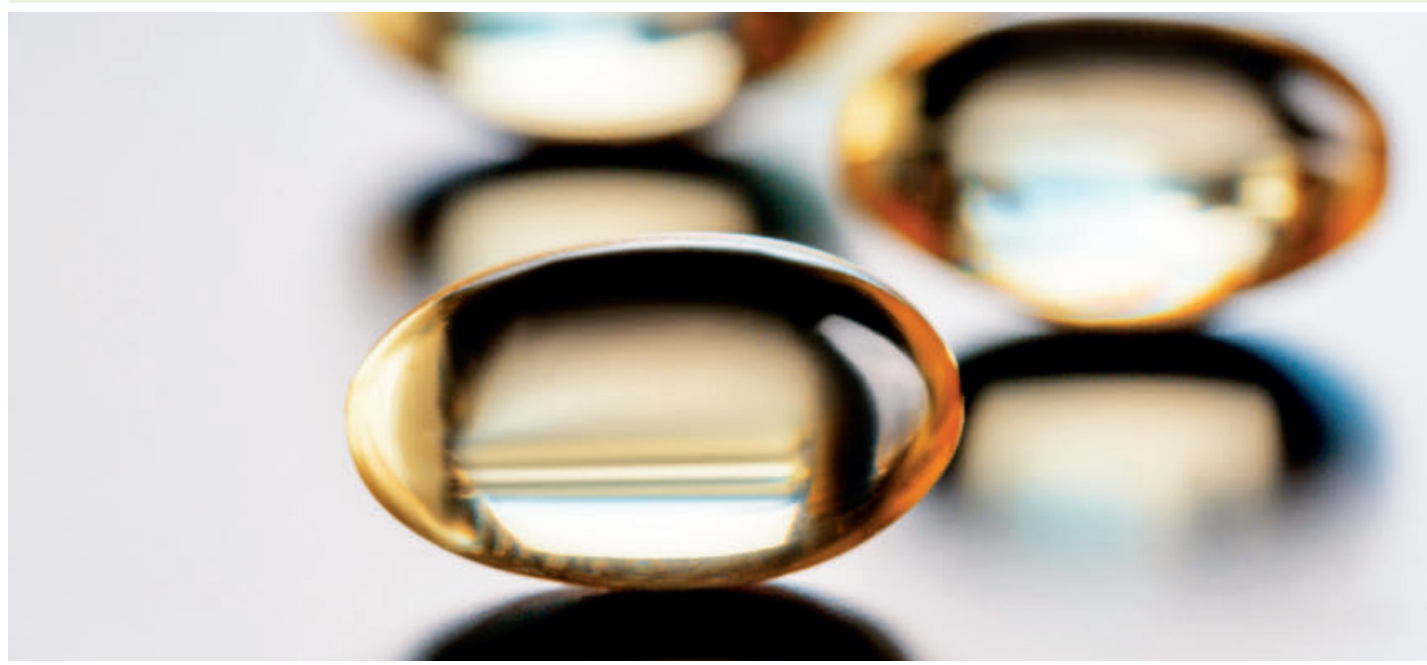

Hochkonzentrierte Fischölkapseln sind geschmacksneutral. @ science photo/Adobe Stock

\section{Silent Inflammation - die stille Gefahr für das Altern}

\begin{abstract}
„Das Alter ist ein kaltes Fieber, im Frost von grillenhafter Not. Hat einer dreißig Jahr vorüber, so ist er schon so gut wie tot. Das Beste wär's, ihn zeitig totzuschlagen. “
\end{abstract}

So lässt der große Dichter und Naturwissenschaftler Goethe seinen Bakkalaureus im Faust sprechen. Stille Entzündung ist tatsächlich so etwas wie „kaltes Fieber“. Eine Übersicht über Inflammageing - den Zusammenhang zwischen Altern und Entzündung - gibt ein aktuelles Review [3] (॰ Abb. 1).
Laut einer Studie ist eine erhöhte Pulswellengeschwindigkeit eindeutig mit stiller Entzündung assoziiert [7]. Diese wird als Maß für die Steifheit, also die fehlende Elastizität, von Gefäßen angesehen. Damit ist sie allgemein als Hinweis für den Grad der Gefäßalterung akzeptiert. Je höher die Pulswellengeschwindigkeit, umso steifer das Gefäß.

Für eine Studie erstellten Wissenschaftler bei 20337 Erwachsenen ohne akute Entzündungszeichen einen Score für stille Entzündungen. Als Biomarker flossen darin ein: CRP-Wert, Leukozytenwert sowie der Quotient aus Granulozyten- und Lymphozytenwert. In 7,6 Jahren kam es zu 837 Todesfällen. Das höchste Quartil des Entzündungsscores wies eine um $44 \%$ höhere Gesamtsterblichkeit im Vergleich zum niedrigsten Quartil auf. Bei Patienten mit Diabetes mellitus Typ II kam es unter denen mit höheren 


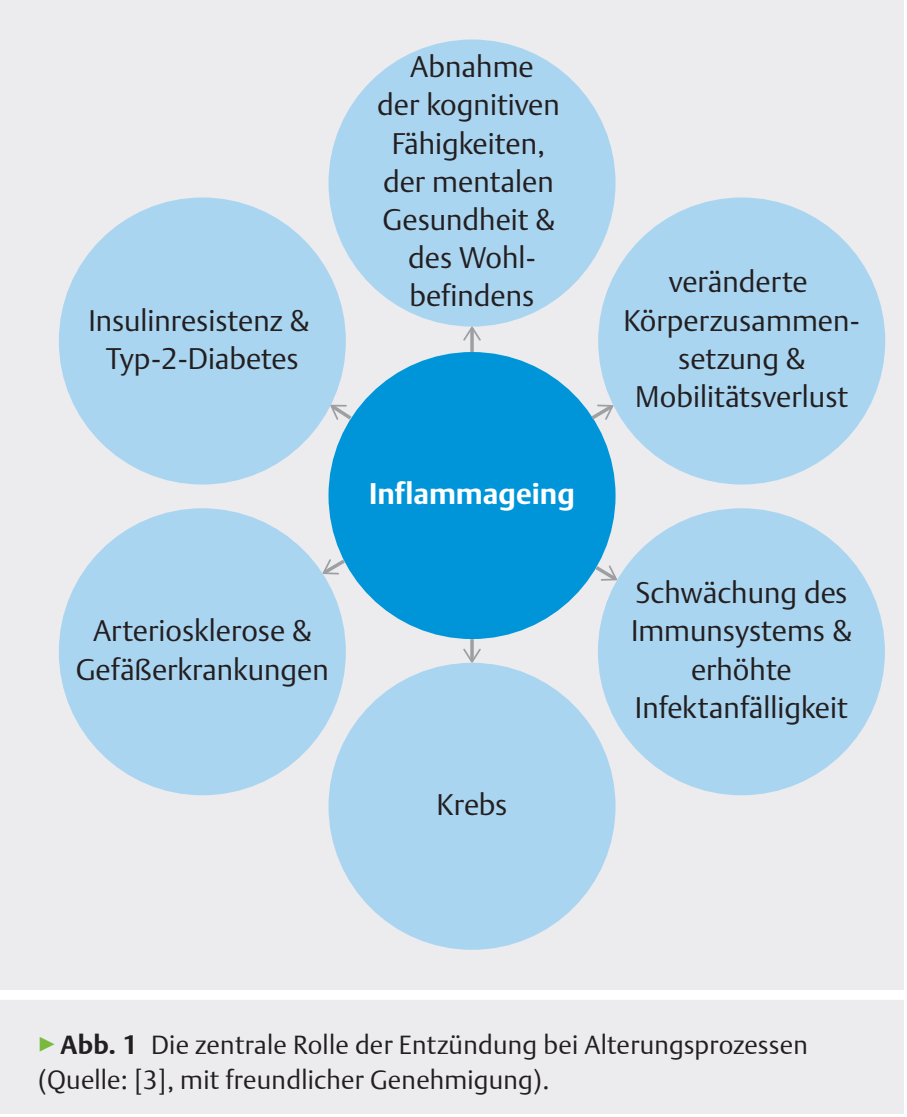

Entzündungsmarkern sogar fast zu einer Verdreifachung $(H R=2,90)$ des Sterberisikos. Bei Patienten mit Koronarer Herzerkrankung (KHK) war das Sterberisiko mehr als verdoppelt $(H R=2,48)$. Stille Entzündungen erwiesen sich damit als signifikanter unabhängiger Risikofaktor für die Gesamtsterblichkeit, besonders bei Risikopersonen [1].

Ein Telomer (griechisch für Endteil) ist eine Nukleotidsequenz am Ende von Chromosomen. Die Telomerenlänge spiegelt das biologische Alter wider. Bei 324 im Jahre 1953 geborenen Versuchspersonen wurden Telomerenlänge, Entzündungsmarker und psychosoziale Ereignisse gemessen. Die Telomeren waren umso kürzer, je mehr Stressereignisse es in der Kindheit gegeben hatte und je höher die Entzündungswerte lagen [11].

\section{Tierversuche}

In einer Studie erhielten Mäuse 3 Monate lang ein Kontrollöl, DHA, eine Mischung aus DHA/DPA (DPA ist eine Intermediärsubstanz zwischen EPA und DHA) oder DHA/AA (Arachidonsäure). Danach wurden im Gehirn der Mäuse Amyloid- $\beta 40$ und Amyloid- $\beta$ 42, die als Marker für Demenz gelten, gemessen. Während die Kontrollgruppe doppelt so hohe Amyloidwerte aufwiesen, lagen alle drei DHA-Gruppen nahezu auf gleichem Niveau. Nach 6 Monaten war das Niveau der Kontrollgruppe weiter angestiegen und lag immer noch doppelt so hoch, wie das Niveau der Gruppen mit DHA oder DHA/DPA (den Omega-3-Gruppen), wäh- rend die DHA/AA-Gruppe sich der Kontrollgruppe angeglichen hatte [6] ( A Abb. 2). Ließe sich dieser Tierversuch auf den Menschen übertragen, würde das bedeuten, dass DHA das Ansteigen von Amyloiden im Alter verzögert. Dieser Effekt würde sich aber nur nachweisen lassen, wenn gleichzeitig keine großen Mengen AA (tierische Fette) konsumiert werden würden.

\section{Demenz - Herausforderung im Alter}

Die Häufigkeit von Demenz nimmt im höheren Lebensalter exponentiell zu. Es scheint, dass die Zahl der Demenzkranken aber nicht nur aufgrund des durchschnittlich steigenden Alters der Menschen zunimmt, sondern mehr Menschen als früher an Demenz erkranken. Eine Erklärung dafür könnten die veränderten Ernährungsgewohnheiten im Allgemeinen und die damit einhergehende Reduzierung von Omega-3-Fettsäuren in der Nahrung im Besonderen sein. In einer Beobachtungsstudie füllten 260 gesunde Probanden einen Ernährungsfragebogen aus, anhand dessen die Zufuhr von Omega-3-Fettsäuren geschätzt wurde. Anschließend wurde bei den Probanden ein Schädel-MRT durchgeführt. Bei der Korrelation verschiedener anatomischer Parameter mit der Ernährung ergab sich eine eindeutige positive Korrelation zwischen Fischkonsum und grauer Hirnsubstanz im orbitalen, frontalen Kortex und dem Hippocampus. Je mehr Fisch die Versuchspersonen verzehrt hatten, umso größer waren die Hirnrinde im beschriebenen Bereich sowie der für Gedächtnisleistungen besonders wichtige Hippocampus [13].

Von 266 Heimbewohnern zwischen 67 und 100 Jahren litten 42 an Demenz, 30 davon an Alzheimer. Der Konsum der Omega-3-Fettsäure Docosahexaensäure (die wichtigste und neben Arachidonsäure die häufigste Fettsäure im Gehirn) wurde anhand eines Fragebogens geschätzt und außerdem der Blutspiegel gemessen. Die Probanden wurden bezüglich ihres DHA-Konsums und ihres DHA-Spiegels in Terzile eingeteilt. Das relative Risiko des Terzils mit den höchsten DHA-Spiegeln betrug für Demenz 0,35 und für Alzheimer 0,40. Die Probanden mit dem meisten DHA in der Nahrung hatten sogar nur ein Risiko von 0,27 für Demenz und von 0,28 für Alzheimer. Eine gute Versorgung mit DHA in der Nahrung konnte in dieser Untersuchung das Risiko also um etwa drei Viertel reduzieren [10].

Die Studie untersuchte auch die Auswirkungen bei bereits vorliegender Demenz. Dafür wurde bei 168 Menschen $\geq 70$ Jahren mit milder kognitiver Störung zu Beginn der interventionellen Studie und zwei Jahre später ein SchädelMRT durchgeführt. Anschließend erhielten die Probanden $0,8 \mathrm{mg}$ Folsäure, $20 \mathrm{mg} \mathrm{B}_{6}, 0,5 \mathrm{mg} \mathrm{B}_{12}$ oder Placebo täglich. Nach zwei Jahren fand sich in der Verumgruppe eine um $40 \%$ geringere Hirnatrophie als Hinweis auf eine neuroprotektive Wirkung der B-Vitamine (vermutlich über verringerte Homocysteinspiegel). Erstaunlich war, dass diese eindrucksvolle Verlangsamung des Hirnabbaus nur 

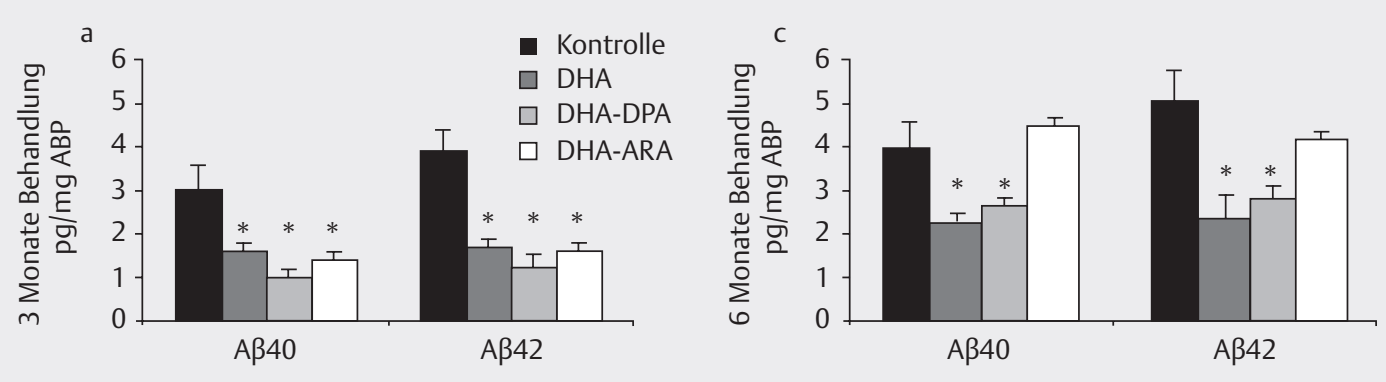

- Abb. 2 Anstieg von Amyloiden unter Zufuhr verschiedener Fette (Quelle: [6]).

bei den Probanden auftrat, die zu Beginn relativ gute Omega-3-Fettsäure-Werte aufwiesen, während bei den Probanden mit schlechter Omega-3-Fettsäure-Versorgung selbst die hoch dosierten B-Vitamine keinen Schutzeffekt entfalten konnten. B-Vitamine schützen also die Nerven, aber nur, wenn Omega-3-Fettsäuren vorhanden sind [8]. Die Gehirnstruktur kann von B-Vitaminen und Omega-3Fettsäuren geschützt werden, was jedoch nicht bedeutet, dass dies auch für die Funktion der Gehirnstruktur gilt. Die Wissenschaftler gingen daher auch dieser Frage nach. 266 Menschen $\geq 70$ Jahren mit milder kognitiver Störung erhielten 2 Jahre lang täglich Vitamin $B_{6}, B_{12}$ und Folsäure in der oben genannten Dosierung oder Placebo. Das Gedächtnis war unter Omega-3-Fettsäuren deutlich besser. DHA erwies sich als effektiver als EPA. Es konnte ebenfalls festgestellt werden, dass die B-Vitamine erst dann richtig wirkten, wenn Omega-3-Fettsäuren vorhanden waren. Die Gruppe mit einer guten Omega-3-Fettsäuren-Versorgung, aber ohne B-Vitamine, hatte sogar bessere Werte als die Gruppe mit schlechter Omega-3-Fettsäuren- und guter Vitamin-B-Versorgung. Erst der Synergismus von Omega-3-Fettsäuren mit B-Vitaminen garantierte die besten kognitiven Werte [12].

In einer weiteren Studie erhielten 485 gesunde Erwachsene mit einem Alter von über 55 Jahren (also im mittleren Alter) und ohne Demenz (Mini-Mental-Status > 26, maximal sind 30 möglich) über 24 Wochen 900 mg DHA oder Placebo. Vorher und nachher wurden kognitive Tests durchgeführt. Nur in der Omega-3-Gruppe ergab sich eine geringere Fehlerhäufigkeit $(p=0,03)$ und ein verbesserter Gedächtnistest $(p=0,02)$. Die Verbesserung der mentalen Fähigkeit entsprach einem 3 Jahre jüngeren Lebensalter kognitiv-mental wirken Omega-3-Fettsäuren also nachgewiesen verjüngend [15].

Der Brain-Derived Neurotrophic Factor (BDNF) ist ein Botenstoff im zentralen Nervensystem, der die Neuroregeneration und Neuroplastizität fördert. Um herauszufinden, ob Omega-3-Fettsäuren BDNF erhöhen können, erhielten 11 Versuchspersonen ohne Kontrollgruppe 12 Wochen lang 1500 mg EPA/DHA. Die Gabe führte zu einem deutlichen durchschnittlichen Anstieg von BDNF [16].

\section{Telomere - lässt sich die biologische Uhr verlangsamen?}

In einer Studie wurde bei 608 ambulanten Patienten mit stabiler KHK eine Messung der Leukozytentelomerenlänge veranlasst. Telomere gelten als biologische Marker für das Altern. Körperzellen können sich nur etwa 50 Mal teilen. Bei jeder Teilung verkürzen sich die Telomeren. Wenn diese aufgebraucht sind, erfolgt die Apoptose, der natürliche Zelltod. Außerdem wurden bei den Patienten EPA und DHA im Blut sowie die Telomerenlänge nach 5 Jahren gemessen. 


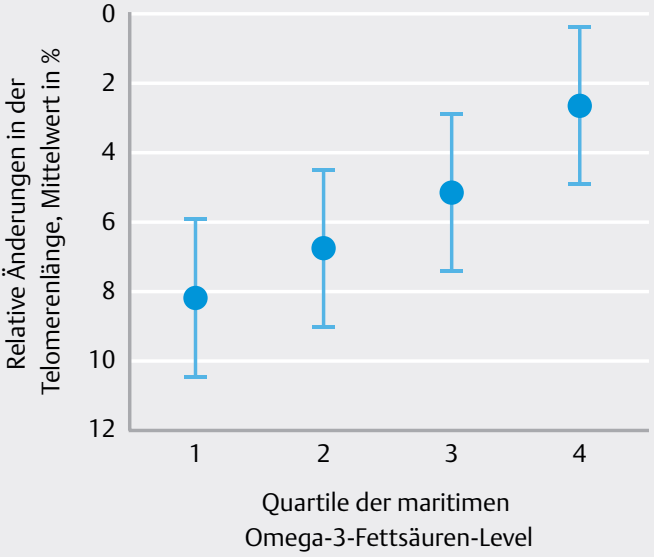

- Abb. 3 Geringere Telomerenverkürzung unter höheren Omega-3-Spiegeln (Quelle: [5]).

Zu Beginn erfolgte eine Einteilung der Patienten in Quartile des Omega-3-Spiegels. Das Quartil mit den höchsten Omega-3-Spiegeln wies am Ende der Studie eine signifikant geringere Telomerenverkürzung $(p<0,001)$ auf. Erstaunlich ist nicht die Signifikanz, sondern das Ausmaß der Verlangsamung der Telomerenverkürzung. Während die Gruppe mit dem niedrigsten Omega-3-Spiegel eine Verkürzung von etwa $8 \%$ zeigte, kam es in der Gruppe mit der besten Omega-3-Fettsäuren-Versorgung zu einer Verkürzung von nur etwa $2 \%$. Anders ausgedrückt: Die Alterung unter dem Aspekt der Telomerenverkürzung - konnte auf ein Viertel reduziert werden [5] ( $\mathbf{A}$ Abb. 3).

\section{Hautalterung und Omega-3-Fettsäuren}

Eine Studie an 2919 gesunden Versuchspersonen im Alter von 45 bis 60 Jahren ging der Frage nach, ob sich Hautfalten, ein biologischer Marker für Alterung, ebenfalls durch Omega-3-Fettsäuren vermindern lassen. Die Probanden füllten einen Ernährungsfragebogen aus, aus dem die Fettsäurezufuhr errechnet wurde. Auf Hautalterung spezialisierte Forscher beurteilten den Hautzustand, ohne die Nahrungszufuhr zu kennen. Dabei zeigte sich, dass die Hautalterung invers zu Omega-3-Fettsäuren korreliert ist. Je niedriger die Omega-3-Fettsäuren-Zufuhr über die Nahrung war, umso mehr und tiefere Hautfalten wurden gefunden [9].

\section{Verlängern Omega-3-Fettsäuren das Leben?}

Zumindest im Tierversuch konnte diese Frage positiv beantwortet werden. Bei schwarzbäuchigen Fruchtfliegen (Drosophila melanogaster) konnte unter Omega-3-Fettsäuren-Zufuhr eine Verlängerung der Lebensdauer von immerhin 14,6\% erzielt werden. Bei den mit Omega-3-Fettsäuren behandelten Fruchtfliegen konnten darüber hinaus eine höhere Elektronentransportkapazität und verbesserte mitochondriale oxidative Kapazität nachgewiesen werden [4]. Ob dieses Ergebnis auf den Menschen übertragbar ist, muss erst noch bewiesen werden. Die Untersuchung zu Telomeren deutet jedenfalls auf ein positives Ergebnis hin.

\section{Muskelschwund im Alter}

Ein großes Problem im Alter ist die Sarkopenie, der Muskelschwund, der mit einem zunehmenden Alter korreliert. Je stärker die Sarkopenie, umso höher ist die Gebrechlichkeit der Patienten. In einem Review wurde der Nutzen von Protein, Vitamin D und Omega-3-Fettsäuren zur Vermeidung von Sarkopenie dargestellt [14]. Eine Metaanalyse mit 36 Studien belegte antiinflammatorische Effekte und die Vermeidung von Sarkopenie bei Älteren. Omega-3Fettsäuren werden in dieser Studie sogar als anaboler Stimulus bezeichnet [2].

\section{Fazit}

Verschiedene Parameter und Symptome des Alterungsprozesses werden durch Omega-3-Fettsäuren günstig beeinflusst. Für Entzündungsbotenstoffe darf dies ebenso als belegt gelten wie für Krankheiten und Symptome wie Demenz, Hautfalten oder Sarkopenie. Vor etwa 10 bis 20 Jahren gab es in der Gesellschaft einen Anti-Aging-Hype. Die positiven Auswirkungen von Omega-3-Fettsäuren waren damals jedoch noch nicht bekannt, da die hier erwähnten Studien fast alle später erfolgten. Hätten die Ergebnisse damals bereits vorgelegen, wären Omega-3-Fettsäuren zu einem der wichtigsten und wirksamsten Mittel apostrophiert worden - im Gegensatz zu vielen damals gemachten Versprechungen.

Wer den Alterungsprozess aufhalten möchte, sollte sich gesund ernähren, viel bewegen und positive Einstellungen beherzigen. Wer außerdem präventiv Omega-3-Fettsäuren in guter Qualität (das Öl darf nicht fischig schmecken!) und Quantität (mindestens $2 \mathrm{~g}$ EPA/DHA, besser nach Fettsäureanalyse: AA/EPA ca. 2,5 und Omega-3-Index $>8 \%$ ) einnimmt, kann das Altern deutlich hinauszögern. Eine physiologisch optimal erreichbare Lebensspanne von 120 Jahren wird sowohl von der 5000 Jahre alten Ayurveda-Medizin als auch von der modernen Gerontologie postuliert. Omega-3-Fettsäuren können dazu beitragen, dem Leben Jahre und den Jahren Leben hinzuzufügen.

Guäti Gsundheit und en langs Läbä! 


\section{Interessenkonflikt}

Der Autor hat in den letzten Jahren Vorträge zur Thematik für die Firmen Biogena, Hepart, Loges und Sanomega gehalten.

\section{Zum Weiterlesen}

Schmiedel V. Omega-3. Öl des Lebens für mehr Gesundheit. Lenzburg: FONA; 2018

Schmiedel V. Vitamine, Mineralstoffe und Spurenelemente: Ernährung, Diagnostik und Nährstofftherapie. Stuttgart: Thieme; 2019

\section{Autor}

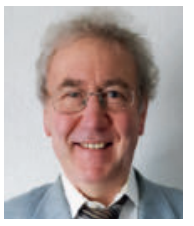

\section{Volker Schmiedel}

war von 1996-2015 Chefarzt der Inneren Abteilung der Habichtswaldklinik Kassel. Seit 2016 ist er als Arzt im ganzheitlichen Ambulatorium Paramed in Baar (Schweiz) tätig. Er war viele Jahre Fortbildungsleiter für „Naturheilverfahren“ der Medizinischen Woche, ist Mitherausgeber der Zeitschrift „Erfahrungsheilkunde" und des "Leitfaden Naturheilkunde“ sowie Autor zahlreicher weiterer naturheilkundlicher Bücher für Therapeuten und Laien.

\section{Korrespondenzadresse}

\section{Dr. med. Volker Schmiede}

Facharzt für Physikalische und Rehabilitative Medizin Naturheilverfahren, Homöopathie

Paramed AC

Haldenstr. 1

CH-6340 Baar

E-Mail: v.schmiedel@paramed.ch

\section{Literatur}

[1] Bonaccio M, Di Castelnuovo A, Pounis G et al. A score of low-grade inflammation and risk of mortality: Prospective findings from the Molisani Study. Haematologica 2016; 101 (11): 1434-1441

[2] Buoite Stella A, Gortan Cappellari G, Barazzoni R et al. Update on the impact of omega 3 fatty acids on inflammation, insulin resistance and sarcopenia: A review. Int J Mol Sci 2018; 19 (1): E218. doi: 10.3390/ijms19010218

[3] Calder PC, Bosco N, Bourdet-Sicard R et al. Health relevance of the modification of low grade inflammation in ageing (inflammageing) and the role of nutrition. Ageing Res Rev 2017; 40: 95-119

[4] Champigny CM, Cormier RPJ, Simard C] et al. Omega-3 monoacylglyceride effects on longevity, mitochondrial metabolism and oxidative stress: Insights from drosophila melanogaster. Mar Drugs 2018; 16 (11): E453. doi: 10.3390/ md16110453
[5] Farzaneh-Far R, Lin J, Epel ES et al. Association of marine omega-3 fatty acid levels with telomeric aging in patients with coronary heart disease. JAMA 2010; 303 (3): 250-257

[6] Green KN, Martinez-Coria H, Khashwji H et al. Dietary docosahexaenoic acid and docosapentaenoic acid ameliorate amyloid-beta and tau pathology via a mechanism involving presenilin 1 levels. J Neurosci 2007; 27 (16): 4385-4395

[7] Greve SV, Blicher MK, Kruger R et al. Elevated estimated arterial age is associated with metabolic syndrome and lowgrade inflammation. J Hypertens 2016; 34 (12): 2410-2417

[8] Jernerén F, Elshorbagy AK, Oulhaj $A$ et al. Brain atrophy incognitively impaired elderly: The importance of long-chain $\omega-3$ fatty acids and $B$ vitamin status in a randomized controlled trial. Am J Clin Nutr 2015; 102 (1): 215-221

[9] Latreille J, Kesse-Guyot E, Malvy D et al. Association between dietary intake of $\mathrm{n}-3$ polyunsaturated fatty acids and severity of skin photoaging in a middle-aged Caucasian population. Dermatol Sci 2013; 72 (3): 233-239

[10] Lopez LB, Kritz-Silverstein D, Barrett Connor E. High dietary and plasma levels of the omega-3 fatty acid docosahexaenoic acid are associated with decreased dementia risk: The Rancho Bernardo study. J Nutr Health Aging 2011; 15 (1): 25-31

[11] Osler M, Bendix L, Rask L et al. Stressful life events and leucocyte telomere length: Do lifestyle factors, somatic and mental health, or low grade inflammation mediate this relationship? Results from a cohort of Danish men born in 1953. Brain Behav Immun 2016 Nov; 58: 248-253

[12] Oulhaj A, Jernerén $F$, Refsum $H$ et al. Omega-3 fatty acid status enhances the prevention of cognitive decline by $B$ vitamins in mild cognitive impairment. J Alzheimers Dis 2016; 50 (2): 547-557

[13] Raji CA, Erickson KI, Lopez OL et al. Regular fish consumption and age-related brain gray matter loss. Am J Prev Med 2014; 47 (4): 444-451

[14] Tessier A], Chevalier S. An update on protein, leucine, omega- 3 fatty acids, and vitamin $\mathrm{D}$ in the prevention and treatment of sarcopenia and functional decline. Nutrients 2018; 10 (8): E1099. doi: 10.3390/nu10081099

[15] Yurko-Mauro K, McCarthy D, Rom D et al. Beneficial effects of docosahexaenoic acid on cognition in age-related cognitive decline. Alzheimers Dement 2010 Nov; 6(6): 456-64. doi: 10.1016/j.jalz.2010.01.013

[16] Matsuoka Y, Nishi D, Yonemoto $\mathrm{N}$ et al. Potential role of brain-derived neurotrophic factor in omega-3 fatty acid supplementation to prevent posttraumatic distress after accidental injury: An open-label pilot study. Psychother Psychosom 2011; 80: 310-312

Bibliografie

DOI https://doi.org/10.1055/a-0828-6082

EHK 2019; 68: 29-33

(c) MVS Medizinverlage Stuttgart GmbH \& Co. KG

ISSN 0014-0082 